\section{Cucumber Germplasm: Isozyme Genetic Stocks W6743, W6744, W6745}

\author{
Jack E. Staub ${ }^{1}$, Vladimir Meglic ${ }^{2}$, Larry D. Knerr², and \\ Linda K. Crubaugh ${ }^{3}$ \\ Department of Horticulture, Vegetable Crops Research, U.S. Department of \\ Agriculture, Agricultural Research Service, University of Wisconsin, Madison, \\ WI 53706
}

Additional index words. Cucumis sativus, allozyme, genetic markers, seed purity assessment, plant variety protection

A series of cucumber (Cucumis sativus L. var. sativus) populations (W6743, W6744, W6745) containing alternate alleles (allozymes) for 20 enzyme coding loci were released in Sept. 1995 by the U.S. Dept. of Agriculture (USDA), Agricultural Research Service. Lines within each population have been created through backcrossing and selfpollination to incorporate allozymes into three genetic backgrounds: European glasshouse, U.S. processing, and U.S. market types. Lines within each population are heterogeneous at specific isozyme or morphological loci and homozygous at others. These genetic stocks could be used for genetic marker research (e.g., linkage assessment, genetic drift detection) or in breeding programs (the incorporation of unique allelic constructs into elite lines) as a tool for varietal purity assessment and plant variety protection.

\section{Origin}

Allelic variation was initially observed in a survey of elite publicly released processing cucumber lines and plant introductions present in the U.S. cucumber collection (USDA Regional Plant Introduction Station, Ames, Iowa) in adenylate kinase (AK), fructose diphosphatase (FDP), glucosephosphate isomerase (GPI), glutathione reductase (GR), glycerate dehydrogenase (G2DH), isocitrate dehydrogenase (IDH), malate dehydrogenase $(\mathrm{MDH})$, manosephosphate isomerase (MPI), peptidase with glycyl-leucine (PEP-GL), peptidase with leucyl-alanine (PEP-LA), peptidase with phenylalanyl-proline (PEP-PAP), peroxidase (PER), phosphoglucomutase (PGM), 6-phosphogluconate dehydrogenase (PGD), and shikimate dehydrogenase (SKDH)

Received for publication 2 Nov. 1995. Accepted for publication 28 July 1996. Mention of a trade name, proprietary product, or specific equipment does not constitute a guarantee or warranty by the U.S. Dept. of Agriculture and does not imply its approval to the exclusion of other products that may be suitable. The cost of publishing this paper was defrayed in part by the payment of page charges. Under postal regulations, this paper therefore must be hereby marked advertisement solely to indicate this fact.

${ }^{1}$ Research Horticulturist and Professor.

${ }^{2}$ Former Graduate Student.

${ }^{3}$ Research Technician.
(Knerr et al., 1989, 1994). Some allozymes of enzymes (e.g., G2DH, IDH, MPI, PGD, PEP$\mathrm{LA}, \mathrm{SKDH}$ ) were not present in elite lines and were recovered from exotic germplasm [e.g., C. sativus var. hardwickii (R) Alef.; PI 183967 and PI 215589; Table 1]. The inheritance of allozymes for these enzymes conforms to Mendelian expectations, and the linkage relationships among these allozymes and between allozymes and some economically important morphological loci have been characterized (Table 2; Knerr and Staub, 1992; Meglic, 1994).

Crosses were made among elite lines, and between elite lines and exotic germplasm to incorporate rare allozymes into adapted germplasm. Lines $\left(>\mathrm{F}_{4}\right)$ were developed and used to determine the inheritance of the isozyme banding patterns resolvable in 15 enzyme systems using horizontal starch gel electrophoresis. These lines (5 to 10 ) were crossed to a European line $\left(\mathrm{F}_{3}\right.$; European glasshouse type $)$ derived from the intermating of three proprietary glasshouse lines from Numhems Zaden BV, De Ruiter Zonen BV, and Nickerson Zwaan, BV, Poinsette 76 (U.S. market type), and GY14 (U.S. processing type). These three lines were used as recurrent parents during backcrossing in which cross progeny heterozygous for isozyme loci were identified and used as parents. After four backcrosses, lines were selfed twice and selected for alternate allozymes at each of 20 loci ( $A k-2, A k-3, F d p$ 1, Fdp-2, Gpi-1, Gr-1, G2dh, Idh, Mdh-1, Mdh-2,Mdh-3,Mpi-1,Mpi-2,Pep-gl,Pep-la, Pep-pap, Per, Pgm, Pgd-1, and Skdh) and uniformity in the three genetic backgrounds. This resulted in the production of six European glasshouse (W6743), 11 U.S. processing (W6744), and eight U.S. market-type (W6745) lines that were homozygous for alternate alleles [e.g., $A k-2$ (11) and (22)] at specific loci (Table 3).

\section{Description}

Although these BC4S2 lines are distinct and can be placed into broad classifications according to potential horticultural utility, they are not phenotypically uniform within a specific type classification (Table 3 ). Lines within a specific type vary in skin texture (smooth or warty) and mature fruit color (green to orange) and length : diameter ratio $(\mathrm{L}: \mathrm{D})$, depending on genetic background. Lines derived using European germplasm as recurrent parents tend to be smooth-skinned, relatively long, and fine-spined. Lines derived from backcrossing to U.S. processing and market lines vary in spine color (black or white) and some segregate for skin texture attributes (i.e., warts and spine thickness) and exhibit a range in $\mathrm{L}: \mathrm{D}$ (2.0 to 4.8). This lack of uniformity could be due to pleiotrophic effects or linkages between allozymes and morphological traits (Meglic, 1994).

\section{Availability}

Limited seed of these genetic stocks are available upon written request to J.E.S.

Table 1. Sources of the less common allozymes in 15 cucumber enzymes. ${ }^{\mathrm{z}}$

\begin{tabular}{|c|c|c|}
\hline Locus $^{y}$ & PI & Source \\
\hline \multirow[t]{2}{*}{$\overline{A k-2(2)}$} & 279469 & Japan \\
\hline & 279463 & Japan \\
\hline \multirow[t]{2}{*}{$A k-3(2)$} & 169334 & Turkey \\
\hline & 255937 & Netherlands \\
\hline \multirow[t]{2}{*}{$F d p-1(2)$} & 169383 & Turkey \\
\hline & 192940 & $\begin{array}{l}\text { People's Republic } \\
\text { of China }\end{array}$ \\
\hline \multirow[t]{2}{*}{$F d p-2(1)$} & 137851 & Iran \\
\hline & 164952 & Turkey \\
\hline \multirow[t]{2}{*}{ Gpi-1(1) } & 200815 & Burma \\
\hline & 422192 & Hungary \\
\hline$G r-1(1)$ & 109275 & Turkey \\
\hline$G 2 d h(1)$ & 285606 & Poland \\
\hline \multirow[t]{2}{*}{$\operatorname{Idh}(1)$} & 215589 & India \\
\hline & 183967 & India \\
\hline \multirow[t]{2}{*}{$M d h-1$ (2) } & 171613 & Turkey \\
\hline & 209064 & United States \\
\hline \multirow[t]{3}{*}{$M d h-2(1)$} & 174164 & Turkey \\
\hline & 357835 & Yugoslavia \\
\hline & 419214 & Hong Kong \\
\hline \multirow[t]{3}{*}{$M d h-3(2)$} & 255236 & Netherlands \\
\hline & 267942 & Japan \\
\hline & 432854 & $\begin{array}{l}\text { People's Republic } \\
\text { of China }\end{array}$ \\
\hline \multirow[t]{2}{*}{$M p i-1(2)^{\mathrm{x}}$} & 176954 & Turkey \\
\hline & 249562 & Thailand \\
\hline \multirow[t]{2}{*}{ Pep-gl(1) } & 113334 & $\begin{array}{l}\text { People's Republic } \\
\text { of China }\end{array}$ \\
\hline & 212896 & India \\
\hline \multirow[t]{2}{*}{ Pep-gl(2) } & 137851 & Iran \\
\hline & 212896 & India \\
\hline \multirow[t]{2}{*}{ Pep-la(2) } & 169380 & Turkey \\
\hline & 354952 & Denmark \\
\hline \multirow[t]{2}{*}{ Pep-pap (1) } & 163213 & India \\
\hline & 188749 & Egypt \\
\hline $\operatorname{Per}(2)$ & 215589 & India \\
\hline \multirow[t]{2}{*}{$\operatorname{Pgm}(2)$} & 171613 & Turkey \\
\hline & 177364 & Iraq \\
\hline \multirow[t]{2}{*}{$P g d-1(1)$} & 169380 & Turkey \\
\hline & 222782 & Turkey \\
\hline \multirow[t]{2}{*}{$P g d-1(2)$} & 188749 & Egypt \\
\hline & 289698 & Australia \\
\hline \multirow[t]{2}{*}{$\operatorname{Skdh}(1)^{\mathrm{x}}$} & 302443 & Taiwan \\
\hline & 390952 & Russia \\
\hline
\end{tabular}

Sources are only given for the less common allele of a locus used based on previous studies by Knerr et al. (1989) and Knerr and Staub (1992). There is also a common allele in addition to each of those listed above.

${ }^{\mathrm{y}}$ Enzyme loci designation, where multiple loci of an enzyme exist, is distinguished by hyphenated numerals and alleles are enclosed in parentheses.

${ }^{x}$ Heterogeneous population containing alternate homozygous and heterozygous genotypes. 
Allelic constitution of enzyme coding loci ${ }^{2}$

\begin{tabular}{|c|c|c|c|c|c|c|c|c|c|c|c|c|}
\hline Identity & $\overline{A k-2}$ & $A k-3$ & $F d p-1$ & $F d p-2$ & Gpi-1 & $G r-1$ & G2dh & $I d h$ & $M d h-1$ & $M d h-2$ & $M d h-3$ & Mpi-1 \\
\hline \multicolumn{13}{|c|}{ European greenhouse type } \\
\hline $6743 \mathrm{~A}$ & 12 & 22 & 22 & 22 & 22 & 11 & 22 & 12 & 22 & 22 & 11 & 12 \\
\hline $6743 B$ & 12 & 22 & 22 & 22 & 22 & 11 & 22 & 12 & 12 & 22 & 11 & 12 \\
\hline $6743 \mathrm{D}$ & 12 & 12 & 12 & 11 & 22 & 11 & 22 & 22 & 11 & 22 & 11 & 12 \\
\hline $6743 \mathrm{E}$ & 22 & 22 & 22 & 22 & 22 & 22 & 11 & 22 & 11 & 22 & 11 & 22 \\
\hline $6743 \mathrm{I}$ & 22 & 22 & 22 & 22 & 22 & 22 & 22 & 22 & 11 & 22 & 11 & 11 \\
\hline $6743 \mathrm{~J}$ & 22 & 22 & 22 & 22 & 22 & 11 & 22 & 22 & 11 & 11 & 11 & 22 \\
\hline \multicolumn{13}{|c|}{ U.S. processing type } \\
\hline $6744 \mathrm{C}$ & 12 & 12 & 22 & 11 & 22 & 11 & 22 & 12 & 11 & 22 & 11 & 22 \\
\hline $6744 \mathrm{~F}$ & 22 & 12 & 12 & 11 & 22 & 11 & 22 & 22 & 22 & 22 & 11 & 22 \\
\hline $6744 \mathrm{G}$ & 22 & 12 & 11 & 22 & 11 & 11 & 22 & 22 & 12 & 22 & 12 & 22 \\
\hline $6744 \mathrm{H}$ & 22 & 12 & 12 & 11 & 22 & 11 & 22 & 22 & 12 & 22 & 22 & 22 \\
\hline $6744 \mathrm{I}$ & 11 & 22 & 22 & 22 & 12 & 11 & 22 & 22 & 11 & 22 & 22 & 22 \\
\hline $6744 \mathrm{~K}$ & 11 & 22 & 22 & 11 & 11 & 11 & 22 & 22 & 11 & 22 & 12 & 22 \\
\hline $6744 \mathrm{~L}$ & 11 & 22 & 22 & 11 & 11 & 11 & 22 & 22 & 11 & 22 & 22 & 22 \\
\hline $6744 \mathrm{M}$ & 11 & 22 & 22 & 11 & 22 & 11 & 22 & 22 & 11 & 11 & 11 & 22 \\
\hline $6744 \mathrm{P}$ & 12 & 11 & 11 & 11 & 22 & 11 & 22 & 22 & 11 & 22 & 11 & 11 \\
\hline $6744 \mathrm{~S}$ & 12 & 12 & 12 & 11 & 22 & 11 & 22 & 22 & 11 & 22 & 11 & 12 \\
\hline $6744 \mathrm{~T}$ & 11 & 12 & 12 & 11 & 22 & 11 & 22 & 22 & 11 & 22 & 11 & 12 \\
\hline \multicolumn{13}{|c|}{ U.S. market type } \\
\hline $6745 \mathrm{~A}$ & 22 & 22 & 11 & 22 & 22 & 11 & 12 & 22 & 12 & 22 & 12 & 11 \\
\hline $6745 \mathrm{D}$ & 22 & 22 & 12 & 22 & 22 & 12 & 22 & 22 & 11 & 22 & 11 & 11 \\
\hline $6745 \mathrm{E}$ & 12 & 22 & 12 & 22 & 22 & 11 & 22 & 22 & 11 & 11 & 22 & 12 \\
\hline $6745 \mathrm{~F}$ & 11 & 22 & 11 & 22 & 22 & 11 & 22 & 22 & 11 & 22 & 12 & 11 \\
\hline $6745 \mathrm{G}$ & 11 & 22 & 11 & 22 & 22 & 11 & 22 & 22 & 11 & 22 & 22 & 11 \\
\hline $6745 \mathrm{H}$ & 12 & 22 & 12 & 22 & 22 & 11 & 22 & 22 & 11 & 12 & 12 & 12 \\
\hline $6745 \mathrm{~J}$ & 12 & 12 & 22 & 11 & 22 & 11 & 22 & 12 & 11 & 22 & 22 & 22 \\
\hline $6745 \mathrm{~K}$ & 11 & 12 & 11 & 11 & 22 & 11 & 22 & 11 & 11 & 22 & 12 & 11 \\
\hline
\end{tabular}

${ }^{2}$ Allozymes that occur in highest frequency are given the mobility designation 100 . All other alleles produce protein products with relative mobilities to allozyme 100 (mm) as follows: Ak-2(1)-98, Ak-3(1)-98, Fdp-1(1)-96, Gpi-1(1)-98, Gr-1(1)-97, G2dh(1)-94, Idh(1)-94, Mdh-1(2)-101.5, Mdh-2(1)-98, Mdh-4(2)-102, Mpi-1(1)-96, Mp-2(2)-103, Pep-gl(1)-98, Pep-la(1)-98, Pep-pap(1)-95, Per(2)-105, Pgm(2)-102.5, Pgd-1(1)-98, Skdh(1)-98.

y Skin texture: $\mathrm{Sm}=$ smooth, $\mathrm{W}=$ warty, $\mathrm{Seg}=$ segregating; skin color: $\mathrm{G}=$ green, $\mathrm{Y}=$ yellow, $\mathrm{O}=$ orange; spine color: $\mathrm{W}=$ white, $\mathrm{B}=$ black, Seg = segregating.

${ }^{\times} \mathrm{L}: \mathrm{D}=$ length $:$ diameter ratio.

Table 2. Enzymes assayed using specific buffer systems that provided adequate resolution of isozyme loci observed in cucumber.

\begin{tabular}{|c|c|c|c|c|}
\hline Enzyme & Abbreviation & $\begin{array}{c}\text { E.C. } \\
\text { designation }^{z}\end{array}$ & $\begin{array}{l}\text { Buffer }^{y} \\
\text { system }\end{array}$ & $\begin{array}{r}\text { No. } \\
\operatorname{loci}^{\mathrm{x}}\end{array}$ \\
\hline Adenylate kinase & $\mathrm{AK}$ & 2.7 .4 .3 & S-4 & 2 \\
\hline Fructose diphosphatase & FDP & 3.1.3.11 & A & 2 \\
\hline Glucosephosphate isomerase & GPI & 5.3 .1 .9 & $\mathrm{R}$ & 1 \\
\hline Glutathione reductase & GR & 1.6.4.2 & S-4 & 1 \\
\hline Glycerate dehydrogenase & G2DH & 1.1.1.29 & $\mathrm{R}$ & 1 \\
\hline Isocitrate dehydrogenase & IDH & 1.1.1.42 & S-4 & 1 \\
\hline Malate dehydrogenase & $\mathrm{MDH}$ & 1.1.1.37 & S-4 & 3 \\
\hline Manosephosphate isomerase & MPI & 5.3 .1 .8 & S-4 & 2 \\
\hline Peptidase with glycyl-leucine & PEP-GL & 3.4.13.11 & A & 2 \\
\hline Peptidase with leucyl-alanine & PEP-LA & 3.4.13.11 & M & 1 \\
\hline Peptidase with phenylalanyl-proline & PEP-PAP & 3.4.13.11 & S-4 & 1 \\
\hline Peroxidase & PER & 1.11.1.7 & A & 1 \\
\hline Phosphoglucomutase & PGM & 5.4 .2 .2 & $\mathrm{R}$ & 1 \\
\hline 6-phosphogluconate dehydrogenase & PGD & 1.1.1.43 & S-4 & 2 \\
\hline Shikimate dehydrogenase & SKDH & 1.1.1.25 & S-4 & 1 \\
\hline
\end{tabular}

${ }^{\mathrm{z}}$ Enzyme commission number.

${ }^{y}$ Buffers of Clayton and Tretiak (1972), Ridgway et al. (1970), and Selander et al. (1971), Markert and Faulhaber (1965) designated as C, R, and S or M, respectively.

${ }^{x}$ Loci designated by previous examination (Knerr and Staub, 1992) or during this survey using standard criteria and nomenclature (Richmond, 1972).

\section{Literature Cited}

Clayton, J.W. and D.N. Tretiak. 1972. Amine-citrate buffers for $\mathrm{pH}$ control in starch gel electrophoresis. J. Fish. Res. Board Can. 29:11691172.

Knerr, L.D., J.E. Staub, D.J. Holder, and B.P. May. 1989. Genetic diversity in Cucumis sativus L. assessed by variation at 18 allozyme coding loci. Theor. Appl. Genet. 78:119-128.

Knerr, L.D.and J.E. Staub. 1992. Inheritance and linkage relationships of isozyme loci in cucumber (Cucumis sativus L.). Theor. Appl. Genet. 84:217-224.

Knerr, L.D., V. Meglic, and J.E. Staub. 1994. A fourth malate dehydrogenase (MDH) locus in cucumber. HortScience 30:118-119.

Market, C.L. and L. Faulhaber. 1965. Lactate dehydrogenase isozyme patterns of fish. J. Expt. Zool. 159:319-332.

Meglic, V. 1994. Inheritance and linkage relationship between biochemical and morphological loci in cucumber (Cucumis sativus L.). $\mathrm{PhD}$ Diss., Univ. of Wisconsin, Madison.

Richmond, R.C. 1972. Enzyme variability in the Drosophila williston group. 3. Amounts of variability in the superspecies $D$. paulistorum. Genetics 70:87-112.

Ridgway, G.L., S.W. Sherburne, and R.D. Lewis. 1970. Polymorphism in the esterase of Atlantic herring. Trans. Amer. Fish. Soc. 99:147-151.

Selander, R.K., M.H. Smith, S.Y. Yang, W.E. Johnson, and J.B. Gentry. 1971. IV. Biochemical polymorphism and systematics in the genus Peromyseus. I. Variation in the old-field mouse (Peromyseus polionotus), p. 49-90. In: M.R. Wheeler (ed.). Studies in genetics. Univ. of Texas Publication, Austin. 


\begin{tabular}{|c|c|c|c|c|c|c|c|c|c|c|c|}
\hline & & & & & & & & \multicolumn{4}{|c|}{ Fruit characteristics ${ }^{y}$} \\
\hline \multicolumn{8}{|c|}{ Allelic constitution of enzyme coding loci ${ }^{2}$} & \multirow{2}{*}{$\begin{array}{l}\text { Skin } \\
\text { texture }\end{array}$} & \multirow{2}{*}{$\begin{array}{c}\text { Skin } \\
\text { color }\end{array}$} & \multirow{2}{*}{$\begin{array}{l}\text { Spine } \\
\text { color }\end{array}$} & \multirow{2}{*}{$\begin{array}{l}\mathrm{L}: \mathrm{D} \\
\text { ratio }^{\mathrm{x}}\end{array}$} \\
\hline Mpi-2 & $P-g l$ & $P$-la & $P$-pap & Per & $P g m$ & $P g d-1$ & $S k d h$ & & & & \\
\hline \multicolumn{12}{|c|}{ European greenhouse type } \\
\hline 11 & 22 & 22 & 22 & 11 & 12 & 22 & 22 & Seg & $G-Y$ to $Y-G$ & W & $3.8: 6.0$ \\
\hline 11 & 12 & 22 & 22 & 11 & 12 & 22 & 22 & Seg & $G-Y$ to $Y-G$ & $\mathrm{~W}$ & $3.8: 6.0$ \\
\hline 12 & 11 & 12 & 22 & 11 & 11 & 12 & 22 & $\mathrm{Sm}$ & $G-Y$ to $Y$ & W & $4.0: 5.5$ \\
\hline 11 & 22 & 22 & 22 & 11 & 11 & 22 & 22 & $\mathrm{Sm}$ & $G-Y$ to $Y$ & W & 3.8:5.6 \\
\hline 11 & 12 & 22 & 22 & 11 & 11 & 22 & 11 & $\mathrm{Sm}$ & $\mathrm{G}$ to $\mathrm{Y}$ & $\mathrm{W}$ & $5.8: 6.0$ \\
\hline 11 & 11 & 22 & 22 & 11 & 22 & 22 & 22 & Seg & $\mathrm{Y}$ & $\mathrm{W}$ & $3.5: 3.8$ \\
\hline \multicolumn{12}{|c|}{ U.S. processing type } \\
\hline 22 & 12 & 22 & 22 & 22 & 12 & 22 & 22 & W & $\mathrm{G}$ to $\mathrm{O}$ & W & $2.8: 3.0$ \\
\hline 22 & 22 & 22 & 22 & 11 & 11 & 22 & 22 & W & $\mathrm{G}-\mathrm{Y}$ to $\mathrm{Y}$ & W & $3.2: 4.0$ \\
\hline 22 & 22 & 12 & 22 & 11 & 11 & 22 & 12 & W & $Y$ to $Y-G$ & W & 3.0:4.5 \\
\hline 22 & 22 & 22 & 22 & 11 & 11 & 22 & 11 & W & $Y$ to $Y-G$ & W & $2.8: 3.5$ \\
\hline 22 & 12 & 22 & 11 & 11 & 11 & 22 & 22 & W & $Y$ to $Y-G$ & W & $2.5: 3.3$ \\
\hline 22 & 22 & 22 & 11 & 11 & 11 & 22 & 22 & Seg & $\mathrm{Y}$ & W & $2.4: 2.8$ \\
\hline 22 & 11 & 22 & 11 & 11 & 11 & 22 & 22 & Seg & Y-G to $\mathrm{Y}$ & W & $2.3: 2.6$ \\
\hline 11 & 11 & 22 & 11 & 11 & 11 & 22 & 11 & Seg & $G-Y$ to $Y$ & W & $2.2: 3.2$ \\
\hline 22 & 11 & 22 & 11 & 11 & 11 & 11 & 22 & W & Y-G to $\mathrm{Y}$ & W & $2.7: 3.5$ \\
\hline 11 & 11 & 22 & 11 & 11 & 11 & 12 & 22 & Seg & $\mathrm{O}$ & B & $2.6: 3.8$ \\
\hline 11 & 11 & 22 & 11 & 11 & 11 & 12 & 22 & Seg & $\mathrm{O}$ to $\mathrm{YG}$ & B & $2.0: 3.5$ \\
\hline \multicolumn{12}{|c|}{ U.S. market type } \\
\hline 12 & 11 & 22 & 22 & 11 & 12 & 22 & 22 & Seg & $\mathrm{O}-\mathrm{Y}$ to $\mathrm{Y}$ & Seg & $3.5: 4.3$ \\
\hline 11 & 12 & 22 & 22 & 11 & 11 & 22 & 12 & Seg & $\mathrm{O}-\mathrm{Y}$ to $\mathrm{Y}$ & Seg & $3.8: 4.3$ \\
\hline 11 & 11 & 22 & 22 & 11 & 12 & 12 & 22 & Seg & G-Y to $Y$ & $\mathrm{~W}$ & $3.3: 3.8$ \\
\hline 11 & 11 & 22 & 22 & 11 & 12 & 11 & 22 & Seg & $\mathrm{G}$ to $\mathrm{Y}$ & $\mathrm{W}$ & $3.0: 3.8$ \\
\hline 11 & 11 & 22 & 22 & 11 & 11 & 11 & 22 & Seg & Y-G to $\mathrm{Y}$ & W & $3.8: 4.5$ \\
\hline 11 & 11 & 22 & 22 & 11 & 12 & 12 & 22 & W & $\mathrm{Y}-\mathrm{G}$ to $\mathrm{Y}$ & $\mathrm{W}$ & $3.8: 4.8$ \\
\hline 11 & 22 & 22 & 22 & 22 & 12 & 22 & 12 & Seg & $\mathrm{Y}$ & B & 3.0:3.8 \\
\hline 22 & 11 & 22 & 22 & 11 & 22 & 22 & 11 & Seg & $\mathrm{O}$ to $\mathrm{Y}$ & B & $3.0: 4.3$ \\
\hline
\end{tabular}

